\title{
Die Entgiftung von zyanidhaltigen Abwässern durch Oxydation mit Hypochlorit
}

\author{
Von W. STUMm, H. WokeR und H. U. Fischer \\ (Aus der Eidgenössischen Anstalt für Wasserversorgung, Abwasserreinigung \\ und Gewässerschutz an der ETH., Zürich, Mitteilung Nr. 84)
}

Eingegangen am 4. März 1954

INHAL'TSVERZEICHNIS

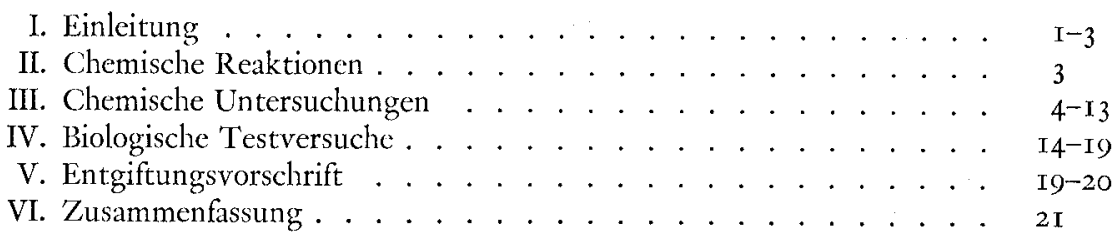

\section{EINLEITUNG}

Wiederholte akute Fischsterben in Vorflutern, denen zyanidhaltige Abwässer aus Metallveredlungsbetrieben oder Härtereien zuflossen, veranlassten uns, die Methoden der sogenannten Zyanidentgiftung nach chemischen und biologischen Gesichtspunkten kritisch zu überprüfen.

Als chemische Behandlungsverfahren für zyanidhaltige Abwässer kommen vor allem folgende Methoden in Betracht:

I. Oxydation des Zyanids mit Chlor beziehungsweise Hypochlorit oder mit Ozon zu Zyanat. Man geht von der Annahme aus, dass dem Zyanat keine nennenswerte Giftwirkung mehr zukomme.

2. Komplexe Bindung des Zyanids an Schwermetallionen (zum Beispiel mit Ferrosulfat). Die an Metalle gebundenen Zyanide haben andere Eigenschaften als das freie Zyanid, und es ist anzunehmen, dass sie kaum schädlich wirken. 
3. Austreiben der mit Mineralsäure in Freiheit gesetzten Blausäure durch Belüften.

Unsere Aufgabe bestand vornehmlich darin, eine betriebstechnisch möglichst einfache und nicht kostspielige Methode auszuarbeiten, die auch chemisch ungeschultem Personal ermöglichen soll, zyanidhaltige Abwässer gefahrlos zu beseitigen. Hier berichten wir über die Behandlung von Zyanidlösungen mit Javellewasser (Hypochlorit). Da die aus galvani-schen Anstalten anfallenden Abwässer fast immer Schwermetalle enthalten, an die das Zyanid komplex gebunden ist, haben wir unsere Untersuchungen auch auf Zyanokomplexe ausgedehnt.

Unsere Arbeiten über Zyanidentgiftung durch Komplexbildung mit Hilfe von Eisensulfat sind noch nicht abgeschlossen. Sie werden in einem spätern Zeitpunkt publiziert werden ${ }^{\mathrm{I}}$ ).

Bezüglich der Wirkung zyanidhaltiger Wässer auf Fische konnten wir uns auf unser eigenes, ziemlich umfangreiches Untersuchungsmaterial stïtzen (WUHRMANN und WOKER, I948, I950, I952, 1953). Es lag deshalb nahe, dass wir zur biologischen Beurteilung der in chemischer Hinsicht als entgiftet zu betrachtenden Lösungen ebenfalls den Fischtest anwandten. Hiefür wurden nach unserer schon mehrfach beschriebenen Versuchstechnik zur Erzielung statistisch gesicherter Versuchsresultate je Io adulte Elritzen (Pboxinus laevis Agas.) in je 61 konstant temperierter Versuchslösung $\left(15^{\circ} \mathrm{C}\right.$ ) bei Sauerstoffsättigung beobachtet. Im Gegensatz zu unseren toxikologischen Untersuchungen verzichteten wir darauf, Konzentrations-Wirkungs-Funktionen für die zu prüfenden Lösungen aufzunehmen. Wir haben lediglich im Sinne einer zusätzlichen analytischen Methode die Wirkungsweise der Entgiftungsverfahren mit einfachen biologischen Versuchen verfolgt. Die Einwirkungszeit der Lösungen auf die Versuchsfische wurde deshalb zunächst auf eine Stunde beschränkt.

Mit der zeitlichen Beschränkung unserer Versuche erfährt allerdings auch der Begriff der Entgiftung eine wesentliche Einengung. Wir haben eine Lösung dann als entgiftet betrachtet, wenn das Verbalten der Testfische innerbalb der ersten 60 min nach Versucbsheginn sicbtbar nicht wesentlich gestört mar. Die vorliegenden Untersuchungen können demnach nicht als eigentliche fischtoxikologische Analysen von Härtereiabwässern oder solchen. aus Galvanisierungsanstalten gelten, denn sie sagen - sofern wir dic Wirkungen nicht noch etwas weiter verfolgt haben - nichts aus über all-

1) Den Herren Dr. F. Zehender und Dr. K. Wunrmann sprechen wir für ihre kritische Sichtung des Manuskripts den besten Dank aus. 
fällig nachträglich auftretende Schädigungen, mit denen unter Umständen zu rechnen ist. Diese Frage erheischt besondere Untersuchungen mit andersgearteter Versuchsanordnung, die wir einer späteren Zeit vorbehalten müssen.

\section{CHEMISCHE REAKTIONEN}

Bei der Oxydation von Zyanid mit Javellewasser spielen sich vornehmlich folgende Reaktionen $a b$ :

$$
\begin{aligned}
& \mathrm{CN}^{-}+\mathrm{OCl}^{-}+\mathrm{H}_{2} \mathrm{O} \longrightarrow \mathrm{ClCN}+2 \mathrm{OH}^{-} \\
& \mathrm{ClCN}^{-} 3 \mathrm{H}_{2} \mathrm{O} \longrightarrow \mathrm{Cl}^{-}+\mathrm{CNO}^{-}+2 \mathrm{H}_{3} \mathrm{O}^{+}
\end{aligned}
$$

Die Reaktion (I) verläuft momentan. Unsere Untersuchungen haben gezeigt, dass die Geschwindigkeit dieser Reaktion sowohl in sauren wie auch in alkalischen Lösungen relativ sehr gross ist. Wir konnten experimentell feststellen, dass bei der Oxydation von $\mathrm{CN}^{-}$mit Hypochlorit immer (auch in stark alkalischen Lösungen) Chlorzyan als Zwischenprodukt gebildet wird. Die Geschwindigkeit der Reaktion (2) ist in starkem Masse von der Hydroxylionenkonzentration abhängig. Prinzipiell kann das in der Reaktion (2) gebildete Zyanat durch zusätzliches Chlor weiteroxydiert werden:

$$
2 \mathrm{CNO}^{-}+3 \mathrm{OCl}^{-}+2 \mathrm{OH}^{-} \longrightarrow 3 \mathrm{Cl}^{-}+\mathrm{N}_{2}+2 \mathrm{CO}_{3}^{--}+\mathrm{H}_{2} \mathrm{O}
$$

Diese Reaktion verläuft ausserordentlich langsam, und sie erfolgt nur dann quantitativ, wenn ein grosser Überschuss an Hypochlorit zur Anwendung gelangt. Für eine Entgiftung zyanidhaltiger Abwässer betrachten wir diese zusätzliche Chlorierung als nicht notwendig, da das in Reaktion (2) gebildete Zyanat keine nennenswerte Giftwirkung mehr aufweist. Eine Überschusschlorierung hätte zudem den Nachteil, dass die Entgiftungsmanipulationen umständlicher würden, indem das verbleibende «freie Chlor» wieder entfernt werden müsste. Andererseits kann das Zyanat vornehmlich in Lösungen von tieferem pH-Wert zu Ammoniumion und Karbonat hydrolysiert werden:

$$
\mathrm{CNO}^{-}+2 \mathrm{H}_{2} \mathrm{O} \longrightarrow \mathrm{NH}_{4}^{+}+\mathrm{CO}_{3}^{--}
$$

Diese Reaktionen können auch in Anwesenheit von Schwermetallen, die Komplexe mit $\mathrm{CN}^{-}$-Ionen bilden, stattfinden (vgl. Seite 5). 


\section{CHEMISCHE UNTERSUCHUNGEN}

I.

Wir haben vorerst abzuklären versucht, ob die Reaktion (I) streng stöchiometrisch verlaufe, das heisst, ob reine Zyanidlösungen, die mit I00\% der theoretisch erforderlichen Menge Hypochlorit versetzt werden, vollständig zyanidfrei seien. Zu Portionen von Lösungen, die verschiedene $\mathrm{CN}^{-}-$Konzentrationen aufwiesen (so zum Beispiel I00, I000, I0000 $\mathrm{mg}$ $\mathrm{CN}^{-} / 1$, wurden verschiedene prozentuale Ansätze der theoretisch erforderlichen Menge Javellewasser zugesetzt. Nach einiger Zeit wurden die verbleibenden Zyanidkonzentrationen bestimmt.

In Figur I sind als Beispiel die Resultate einer Versuchsserie eingetragen. Die Experimente haben ergeben, dass bei einer Zugabe von 100\% der

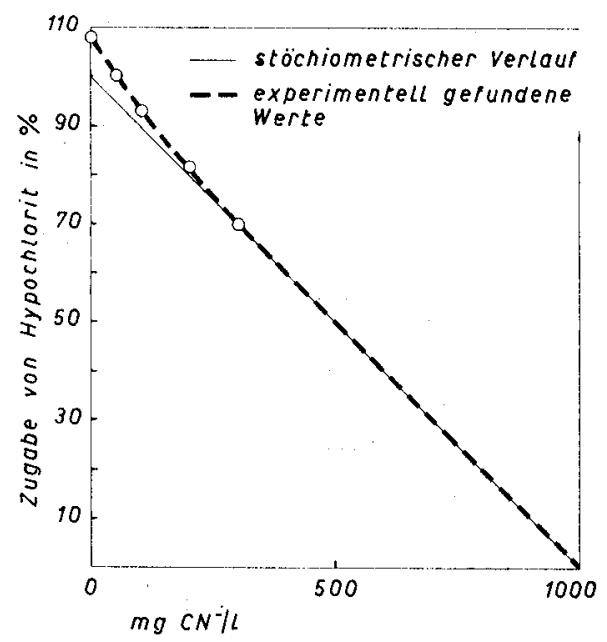

Figur 1. Umsatz von Zyanid mit Hypochlorit.

theoretisch erforderlichen Menge von OCl- immer noch 2-4\% der ursprünglich vorhandenen $\mathrm{CN}^{-}$-Konzentration nicht oxydiert wurden. Es scheint, dass die Reaktion stöchiometrisch verläuft bis zu einer Stufe, bei der die $\mathrm{CN}^{-}$-Ionenkonzentration gegenüber der Konzentration an gebildetem Zyanat sehr klein geworden ist. Man muss annehmen, dass dann neben Reaktion (I) das schon gebildete Zyanat zum Teil weiteroxydiert wird, wie es in Gleichung (3a) formuliert ist. Unsere Versuche haben gezeigt, dass neben einem noch so kleinen Úberschuss an «freiem Chlor» 
$\mathrm{CN}^{-}$-Tonen nicht beständig sind. In Lösungen, deren $\mathrm{pH}$ unter 8 liegt, kann das gebildete $\mathrm{CNO}^{-}$zu einem Teil zu Ammoniumverbindungen verseift werden [Reaktion ( $3 \mathrm{~b})$ ], was sich bei der Javellebehandlung oft durch die Bildung von Chloraminen bemerkbar macht. Aber auch bei der alkalischen Oxydation von konzentrierten Zyanidlösungen mit Javellewasser können Spuren von Ammoniak entstehen.

\section{Die Oxydation von Zyanometallkomplexen mit Hypochlorit}

Die aus der Galvanotechnik anfallenden Zyanidbäder enthalten fast ausnahmslos Schwermetalle, die mit dem Zyanid lösliche Komplexe bilden. Die wichtigsten dabei auftretenden Zyanokomplexe sind:

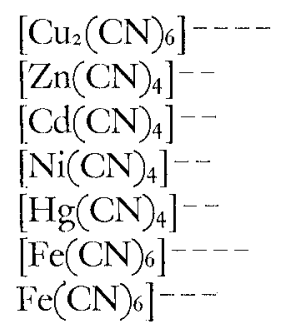

[Hexazyanodikuprat(I)]
[Tetrazyanozinkat]
[Tetrazyanokadmiat]
$[$ Tetrazyanonickolat(II)]
$[$ Tetrazyanomerkurat(II)]
$[$ Hexazyanoferrat(II)]
$[$ Hexazyanoferrat(III)]

In Bädern, die komplexe Zyanide enthalten, ist zu unterscheiden zwischen der Konzentration an Gesamtzyanid

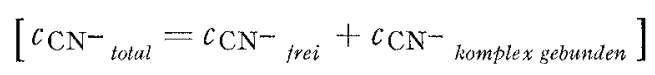

und der Konzentration an freiem Zyanid

$$
\left[\mathrm{CNN}^{-}{ }_{f r e i}\right] \text {. }
$$

Die Konzentration an freiem Zyanid ergibt sich einerseits aus $c_{\mathrm{CN}^{-}}{ }_{\text {toial }}$ und aus der Konzentration an komplex gebundenem Metall, andererseits aus der Stabilität und der Robustheit des Zyanometallkomplexes. Die Stabilität des Metallkomplexes lässt sich aus den entsprechenden Komplexdissoziationskonstanten abschätzen.

$$
\begin{gathered}
{\left[\mathrm{Me}^{+m}\left(\mathrm{CN}^{-}\right)_{x}\right]^{(m-x)} \cdots \mathrm{Me}^{+m}+x\left(\mathrm{CN}^{-}\right),} \\
K_{D}=\frac{\left(\mathrm{Me}^{+m}\right)\left(\mathrm{CN}^{-}\right)^{x}}{\left(\left[\mathrm{Me}^{+m}\left(\mathrm{CN}^{-}\right)_{x}\right]^{(m-x)}\right)} ;(\ldots) \text { bedeuten Konzentrationen. }
\end{gathered}
$$

Die Grössenordnungen einiger Dissoziationskonstanten sind in Tabelle I aufgezcichnet. Die dort erwähnten Komplexe sind in der Reihenfolge ab- 
Tabelle 1

\begin{tabular}{|c|c|c|}
\hline Komplex & $\begin{array}{c}K_{D} \\
\text { (nach MILNE 1950) }\end{array}$ & $\begin{array}{l}\frac{\left(\mathrm{CN}^{-}\right)_{f r e i} \text { in } \gamma / 1}{\text { bei Vorliegen eines stöchiometrisch }} \\
\text { reinen Komplexes der Konzentration } \\
\mathrm{CN}^{-} \text {total }=1000 \mathrm{mg} / 1\end{array}$ \\
\hline $\begin{array}{l}{\left[\mathrm{Hg}(\mathrm{CN})_{4}\right]^{--}} \\
{\left[\mathrm{Ag}(\mathrm{CN})_{2}\right]^{-}} \\
{\left[\mathrm{Cu}{ }_{2}(\mathrm{CN})_{6}\right]^{-}} \\
{\left[\mathrm{Fe}(\mathrm{CN})_{6}\right]^{---}} \\
{\left[\mathrm{Ni}(\mathrm{CN})_{4}\right]^{--}} \\
{\left[\mathrm{Cd}(\mathrm{CN})_{4}\right]^{--}} \\
{\left[\mathrm{Zn}(\mathrm{CN})_{4}\right]^{--}}\end{array}$ & $\begin{array}{r}4 \cdot 10^{-42} \\
1 \cdot 10^{-21} \\
5 \cdot 10^{-28} \\
1 \cdot 10^{-36} \\
1 \cdot 10^{-22} \\
1,3 \cdot 10^{-17} \\
1,4 \cdot 10^{-17}\end{array}$ & $\begin{array}{c}0,07 \\
0,9 \\
8,1 \\
116 \\
540 \\
\mathbf{5 7 0 0} \\
\mathbf{5 7 6 0}\end{array}$ \\
\hline
\end{tabular}

nehmender Stabilität aufgeführt. 'Zur Illustration ist in der dritten Kolonne diejenige Konzentration an freiem Zyanid angegeben, die in Lösungen stöchiometrisch reiner Metallkomplexe mit einer Totalzyanidkonzentration von $1000 \mathrm{mg} / \mathrm{l}$ vorhanden ist. Vom chemischen Standpunkte aus war vorerst abzuklären, wie das komplex gebundene Zyanid sich gegenüber Hypochlorit verhält. Dem biologischen Versuch blieb es dann vorbehalten, zu untersuchen, welche Zyanometallkomplexe genügend stabil sind, um innert der von uns gewählten Versuchsbedingungen keine Giftwirkung mehr hervorzurufen. Die Übersicht über die Stabilitätsverhältnisse (vgl. Tabelle I) zeigt, dass Lösungen von Zyanozinkat- und Zyanokadmiatkomplexen eine $z u$ hohe Konzentration an freiem Zyanid aufweisen, um gemäss unserer Kenntnisse «giftfrei» zu sein. Die relativ schwache Komplexbildungstendenz von Zink und Kadmium zu Zyanid erklärt, dass in solchen Lösungen - im Gegensatz zu den anderen Schwermetallkomplexen - auch das gebundene $\mathrm{CN}^{-}$ohne Schwierigkeiten mit $\mathrm{AgNO}_{3}$ titriert werden kann. Die Oxydation von Lösungen solcher Zyanometallverbindungen mit Hypochlorit gelingt erwartungsgemäss bei weniger stabilen Komplexen besser als bei solchen, die eine kleine Dissoziationskonstante aufweisen. Ein Ausschnitt unserer experimentellen Versuche findet sich in Tabelle 2. Die dort aufgeführten Werte illustrieren deutlich die verschiedenen Stabilitätsverhältnisse der Komplexe. Bei der Oxydation des Hexazyanoferrates (II) mit Hypochlorit, muss berücksichtigt werden, dass vorgängig der Zersetzung der Verbindung eine Oxydation zu dem ausserordentlich robusten Hexazyanoferrat(III)-Komplex stattfindet. Von den untersuchten Komplexen lässt sich für die Kadmium-, Zink- und Nickelverbindungen das gebundene Zyanid ohne weiteres zu Zyanat oxydieren, 
Tabelle 2

\begin{tabular}{|c|c|}
\hline Komplex & $\begin{array}{c}\left(\mathrm{CN}^{-}\right)_{\text {total }} \text { in Prozent des ursprünglich vorhandenen } \\
\left(\mathrm{CN}^{-}\right)_{\text {total }} \text { nach Zugabe von } 100 \% \text { der zur } \mathrm{CN}^{-} \text {-Oxydation } \\
\text { theoretisch erforderlichen Hypochloritmenge }\end{array}$ \\
\hline $\mathrm{KCN}$ & 3 \\
{$\left[\mathrm{Cd}(\mathrm{CN})_{4}\right]^{\cdots \cdots-}$} & 3 \\
{$\left[\mathrm{Zn}(\mathrm{CN})_{4}\right]^{--}$} & 3,5 \\
{$\left[\mathrm{Ni}(\mathrm{CNN})_{4}\right]^{--}$} & 6 \\
{$\left[\mathrm{Hg}(\mathrm{CN})_{4}\right]^{--}$} & 30 \\
{$\left[\mathrm{Fe}(\mathrm{CN})_{6}\right]^{--}$} & 87 \\
\hline
\end{tabular}

wobei aber stets ein kleiner Überschuss an Hypochlorit angewendet werden muss. Bei der Oxydation des Nickelkomplexes entsteht bei Zugabe von überschüssigem Hypochlorit das schwarze Nickel(III)-hydroxyd.

\section{Die Verseifung von Chlorzyan}

Die Geschwindigkeit der Reaktion (2) ist in ausserordentlich starkem Masse von der Hydroxylionenkonzentration abhängig.

$\mathrm{Zu}$ Lösungen, die jeweils to $\mathrm{mg} \mathrm{CN}-1$ enthielten und die mit Soda, Bikarbonat oder Azetat gepuffert waren, wurden je 100\% der theoretisch erforderlichen Menge OCl- zugegeben. Zu verschiedenen Zeiten nach der Javellezugabe wurde die ClCN-Konzentration photometrisch gemessen. Unsere Resultate sind in Figur 2 aufgezeichnet. In Lösungen von $\mathrm{pH}=7$ oder darunter ist innert $24 \mathrm{~h}$ die $\mathrm{ClCN}^{-}$-Konzentration höchstens um die Hälfte zurückgegangen. Auch bei $\mathrm{pH}=7,95$ ist Chlorzyan nach $6 \mathrm{~h}$ noch deutlich nachweisbar. Sogar in Lösungen des $\mathrm{pH}=9,2$ beträgt der ClCN-Gehalt nach $2 \mathrm{~h}$ noch etwa $15 \%$ des ursprünglich vorhandenen Wertes. Die ClCN-Verseifung ist bei $\mathrm{pH}=10,85$ innert $45 \mathrm{~min}$, bei $\mathrm{pH}=\mathrm{I} 2,5$ innert $20 \mathrm{~min}$ vollständig. Diese Resultate illustrieren, dass es für die Entgiftung von zyanichaltigen Abwässern durch Oxydation mit Chlor unbedingt notwendig ist, auch während des Oxydationsverfahrens den pH zu kontrollieren, wobei man berücksichtigen muss, dass in der Reaktion (I) - (2) das $\mathrm{CN}^{-}-\mathrm{Ion}$, das eine starke Base ist, verschwindet und dass sich dadurch der $\mathrm{pH}$ eines nur schwach gepufferten Abwassers (trotz der mit dem Javellewasser zugegebenen Lauge) erniedrigt.

Chlorzyan ist sehr flüchtig und speziell in gasförmiger Form für den Menschen von hoher Giftigkeit, so dass es auch aus diesem Grunde wünschbar ist, die Entgiftungsreaktion durch Regulierung des $\mathrm{pH}$ so 
durchzuführen, dass eine möglichst schnelle Verseifung des ClCN stattfindet. Die Reaktion (I) - (2) ist exotherm, bei Javellezugabe steigt die Temperatur der zu entgiftenden Lösung stark an. Um die Verflüchtigung von Chlorzyan einzuschränken, ist zu vermeiden, dass die Temperatur

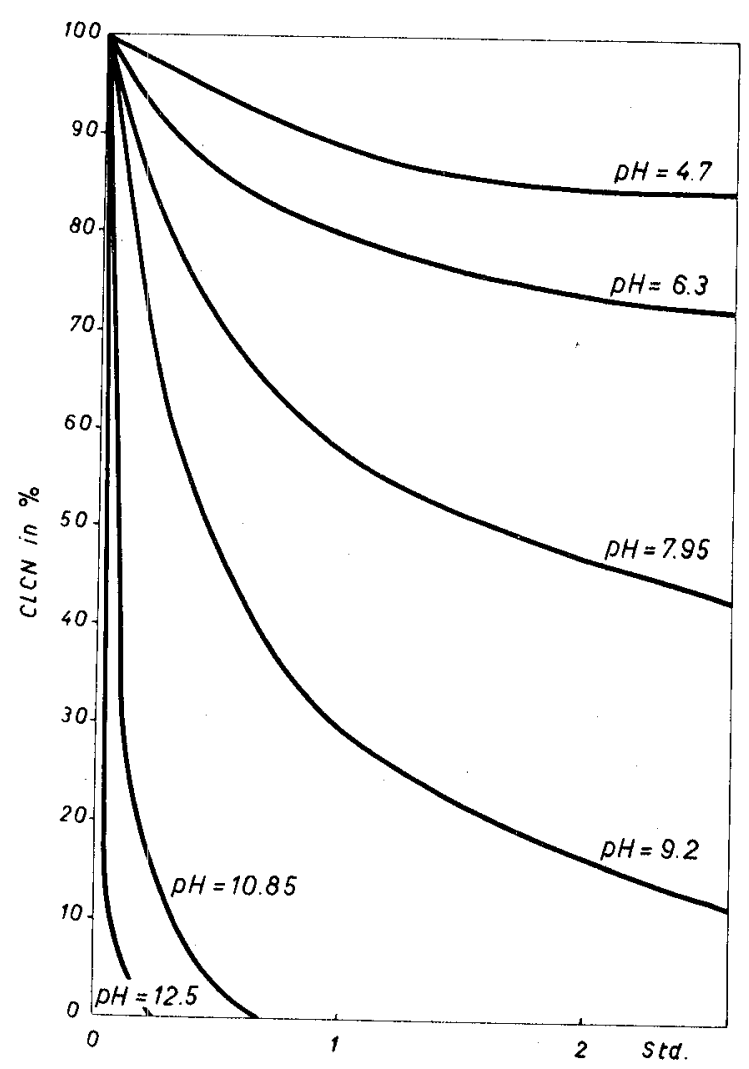

Figu: 2. Geschwindigkeit der Hydrolyse von Chlorzyan in Abhängigkeit vom pH.

der Lösung, bei der die Entgiftungsoperation erfolgt, $50^{\circ} \mathrm{C}$ übersteigt. Nach unseren Erfahrungen kann die Oxydation von Zyanid mit Javellelauge bei Bädern, die bis zu tooo $\mathrm{mg} \mathrm{CN}-/ 1$ enthalten, durchgeführt werden, ohne dass die Temperatur $50^{\circ} \mathrm{C}$ übersteigt, wenn die Javellezugabe langsam und unter stetem Umrühren erfolgt. Unsere Versuche über die Geschwindigkeit der Hydrolyse von $\mathrm{ClCN}$ lassen es wünschbar erscheinen, dass die Oxydation von Zyanid mit Hypochlorit bei einem möglichst hohen $\mathrm{pH}$ durchgeführt werde. Dem steht aber entgegen, dass «ent- 
giftete» Bäder mit pH-Werten oberhalb 9 ohne Neutralisierung aus biologischen Gründen nicht dem Vorfluter übergeben werden sollen. Die Neutralisierung mit einer Säure hat aber zur Folge, dass bei der Säurezugabe die Reaktion ( 3 b) (die Verseifung von Zyanat zu $\mathrm{NH}_{4}{ }^{+}$) gefördert wird. Das Entstehen von Ammoniak bei der Säurezugabe kann indessen weitgehend verhindert werden, wenn die Neutralisierung mit einer nicht allzu konzentrierten Säure $(<5 \%$ ) langsam und unter Umrühren erfolgt. Es ist vorteilhaft, wenn dabei der $\mathrm{pH}$ nur auf 8,5 bis 9 erniedrigt wird.

\section{4.}

Eine Lösung, in der das Zyanid mit Hypochlorit oxydiert worden ist, kann, chemisch gesehen, nur dann als « entgiftet»betrachtet werden, wenn einerseits das ausserordentlich giftige Chlorzyan nicht mehr nachgewiesen werden kann und wenn andererseits die Lösung kein «freies Chlor» enthält. Ferner darf das Wasser höchstens Spuren von Ammoniumionen enthalten und soll einen $\mathrm{pH}$ unter 9 aufweisen. Dementsprechend müssen vom chemischen Standpunkte aus vor allem folgende Forderuhgen für die Entgiftung erhoben werden:

a) Der $\mathrm{pH}$ der zu entgiftenden Lösung darf auf jeden Fall nicht unter 8 liegen. Auch während des Oxydationsvorganges soll ein pH-Wert von 8 nicht unterschritten werden. (Begründung: Unterhalb $\mathrm{pH}=8 \mathrm{er}$ folgt die Hydrolyse von ClCN zu Zyanat zu langsam. Zudem wird ein Teil des gebildeten Zyanats in $\mathrm{NH}_{4}{ }^{+}$umgewandelt. Ausserdem besteht die Gefahr von Blausäureentwicklung.)

b) Bei der Entgiftungsoperation darf die Temperatur der Lösung $50^{\circ} \mathrm{C}$ nicht übersteigen. (Begründung: Verflüchtigung von ClCN.)

c) Die zum Zyanidabwasser zuzugebende $\mathrm{OCl}^{--}$-Menge muss in jedem einzelnen Fall experimentell bestimmt werden. (Begründung: Es wird mehr als I00\% der theoretisch erforderlichen Menge Javellewasser zur Oxydation des $\mathrm{CN}^{-}$-haltigen Abwassers benötigt; je nach dem Chlorzehrungsvermögen des Abwassers wird noch zusätzlich « freies Chlor» gebunden.)

d) Nach der Chlorzugabe muss so lange gewartet werden, bis alles ClCN hydrolysiert ist.

$\begin{array}{cc}\mathrm{pH} \text {-Wert nach Javellezugabc } & \text { Wartefrist } \\ 8 & 20 \mathrm{~h} \\ 9 & \text { I2 h } \\ \text { IO } & 4 \mathrm{~h} \\ \text { II } & \text { I h } \\ \text { I2 } & 0,25 \mathrm{~h}\end{array}$


e) Eine allfällige Neutralisation darf nur bis hinunter $\mathrm{zu} \mathrm{pH}=8,5-9$ erfolgen. Zur Neutralisation ist eine möglichst wenig konzentrierte Säure zu verwenden. Die Säurezugabe muss langsam und unter Umrühren erfolgen. (Begründung: $\mathrm{NH}_{3}$-Bildung aus Zyanat.)

f) Das zu behandelnde Wasser soll auf keinen Fall mehr als $1000 \mathrm{mg} \mathrm{NO}_{3}$ pro Liter enthalten. (Begründung: Bildung von giftigem Nitrosylchlorid.)

5.

Unsere Experimente haben gezeigt, dass eine solche Oxydation von Zyanid in industriellen Abwässern mit Hilfe von Hypochlorit ohne Schwierigkeiten durchgeführt werden kann, wenn die zu entgiftenden Lösungen Zyanidkonzentrationen aufweisen, die kleiner als etwa Iooo $\mathrm{mg}$ $\mathrm{CN}^{-} / 1$ sind. In konzentrierteren Lösungen wird die $\mathrm{NH}_{3}$-Konzentration [vgl. Reaktion (3b)] zu gross. Beispielsweise betrug die $\mathrm{NH}_{3}$-Konzentration einer mit Javellelauge oxydierten Lösung, die $5000 \mathrm{mg} \mathrm{CN}-/ 1$ enthielt, I80 $\mathrm{mg} \mathrm{NH} / 1$. Abwässer, deren Zyanidgehalt grösser als $1000 \mathrm{mg}$ $\mathrm{CN}^{-} / 1$ ist, müssen deshalb, wenn sie mit Hypochlorit entgiftet werden sollen, vorerst entsprechend verdünnt werden.

\section{Analytisches. Zyanidbestimmung}

\section{a) Durch Titration mit Silbernitrat}

Diese von LIEBIG beschriebene Methode beruht auf der Bildung des löslichen und ausserordentlich stabilen Dizyanosilberkomplexes:

$$
{ }_{2} \mathrm{CN}^{-}+\mathrm{Ag}^{+} \longrightarrow\left[\mathrm{Ag}(\mathrm{CN})_{2}\right]^{-} \text {. }
$$

Wenn man bei der Titration mehr Ag+-Ionen zufügt, als zur Bildung des löslichen Komplexes notwendig sind, so wird schwerlösliches $\{\mathrm{AgCN}\}$ ausgefällt:

$$
\left[\mathrm{Ag}(\mathrm{CN})_{2}\right]^{-}+\mathrm{Ag}^{+} \longrightarrow 2\{\mathrm{AgCN}\}
$$

Der Äquivalenzpunkt kann dabei durch die von $\{$ AgCN $\}$ hervorgerufene Trübung erkannt werden. Eine bessere Indikation des Titrationspunktes wird erreicht, wenn man nach dem Vorschlag von KoLTHOFF dem zu titrierenden Wasser KJ zufügt. Der Umschlagspunkt ist dann erreicht, wenn gelbes Silberjodid auszufallen beginnt. FEIGL hat zum qualitativen Nachweis von Silberionen Dimethylaminobenzylidenrhodanin als emp- 
findlichen Indikator vorgeschlagen. Dieser Farbstoff eignet sich vorzüglich bei der Titration von Zyanid mit Silbernitrat als Indikator. Nach

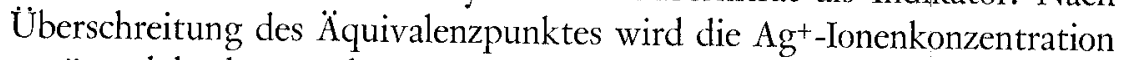
genügend hoch, um den rotgefärbten Silber-Dimethylaminobenzylidenrhodanin-Komplex zu bilden.

Ausfübrung: Die zu untersuchende Lösung wird auf einen $\mathrm{pH}$-Wert von $\sim$ ro gebracht (zum Beispiel mit Soda) und mit einigen Tropfen einer alkalischen Lösung von Dimethylaminobenzylidenrhodanin versetzt. Mit Silbernitrat wird bis zum Farbumschlag (gelb-orangerot) titriert.

Das direkte Titrationsverfahren spricht spezifisch auf $\mathrm{CN}^{-}$-Ionen an. [Die Reaktion wird nur gestört, wenn in der Lösung noch andere Substanzen enthalten sind, welche mit Silber Komplexe bilden können (zum Beispicl Thiosulfat, Sulfide).]

Der Farbumschlag bei der Titration ist sehr scharf, so dass mit sehr verdünnten $\mathrm{AgNO}_{3}$-Lösungen titriert werden kann (zum Beispiel: $m /$ roo). Die Methode gestattet, eine $\mathrm{CN}^{-}$-Bestimmung mit einer Genauigkeit von $\pm 0, \mathbf{I} \mathrm{mg} \mathrm{CN-/1}$ ohne Schwierigkeiten durchzuführen.

Obschon diese Titrationsart in unserem Institut seit Jahren gebräuchlich ist, wurde sie unseres Wissens erstmals von WILL (I95iI) erwähnt.

\section{b) Kolorimetrische Methoden}

Oft ist es hingegen wünschbar, sehr geringe Zyanidkonzentrationen $(<\mathrm{I} \mathrm{mg} \mathrm{CN-/1)} \mathrm{zu} \mathrm{analysieren.} \mathrm{Dazu} \mathrm{eignen} \mathrm{sich} \mathrm{vor} \mathrm{allem} \mathrm{kolorimetri-}$ sche Methoden. Sehr empfindlich ist zum Beispiel die Farbreaktion, die Aloin mit Zyanidionen in Gegenwart von Kupfer zeigt.

Verfabren: Reagenzien: Aloinlösung, 0,5prozentig, in Alkohol; Kupfersulfatlösung, Iprozentig in Wasser; Zitratpuffer, pH 6,5, 0,2 smolar.

Ausfübrung: $\mathrm{Zu} 100 \mathrm{~cm}^{3}$ der $\mathrm{zu}$ untersuchenden Lösung gibt man $0,5 \mathrm{~cm}^{3}$ Kupfersulfat und nach kurzem Durchmischen $5 \mathrm{~cm}^{3}$ Aloinlösung. Nach ungefähr zwei Minuten setzt man $5 \mathrm{~cm}^{3}$ Zitratpuffer zu und mischt nochmals gut durch. In Anwesenheit von Zyaniden entsteht sofort eine Rosafärbung, die während rund 30 min unverändert bleibt. Für lichtelektrische Messungen empfiehlt sich die Wellenlänge von $550 \mathrm{~m} \mu$. Dieses kolorimetrische Bestimmungsverfahren erlaubt Zyanidbestimmungen bis hinunter zu Konzentrationen von $0,02 \mathrm{mg} \mathrm{CN}-11$ sicher durchzuführen. Unsere Untersuchungen haben aber gezeigt, dass diese Bestimmungsmethode folgende Nachteile aufweist: Eine analoge Farbreaktion wird durch $\mathrm{CNO}^{-}$- und $\mathrm{CNS}^{-}$-Ionen hervorgerufen, was diese 
Methode als unspezifisch für unsere Zwecke ausschliesst. Es ist oft schwierig, den für die Reaktion erforderlichen $\mathrm{pH}$ genügend genau einzuhalten, was für reproduzierbare Bestimmungen unbedingt erforderlich ist. In neuerer Zeit ist eine spezifische, kolorimetrische Methode beschrieben worden (ALDRIDGE, I944; EPSTEIN, I947), die auf folgendem Prinzip basiert (KÖNIG, I9O4): Bei der Zugabe von Brom zu einer Zyanidlösung entsteht Bromzyan, das in Gegenwart von primären oder sekundären aromatischen Aminen auf Pyridin so einwirkt, dass unter Aufspaltung des Pyridinringes ein intensiv gefärbtes Dianilid des Glutakondialdehyds entsteht.

Wir haben mit folgendem Analysenverfahren, das wir nach einer Anregung von AsMUS (I952) modifiziert haben, gute Resultate erhalten: Io $\mathrm{cm}^{3}$ des zu untersuchenden Wassers werden mit I $\mathrm{cm}^{3}$ eines 5 molaren Essigsäure-Azetatpuffers von $\mathrm{pH}=5$ versetzt. $0,5 \mathrm{~cm}^{3}$ Bromwasser werden zugegeben und die Lösung kurz umgeschüttelt. Dazu werden hintereinander ohne Zeitverlust in folgender Reihenfolge zugesetzt:

I. $0,5 \mathrm{~cm}^{3}$ einer 2 prozentigen Arsenitlösung;

2. $6 \mathrm{~cm} 3$ eines jeweils frisch hergestellten Mischreagens (Zusammensetzung: I g Benzidinhydrochlorid in $0,2 \mathrm{~m}$ Salzsäure ( $\left.15 \mathrm{~cm}^{3}\right)+15 \mathrm{~cm}^{3}$ Pyridin);

3. $4 \mathrm{~cm}^{3}$ Azeton.

Nach kräftigem Umschütteln bleibt die Lösung 30 min stehen. Die Intensität der Farbe kann bei $530 \mathrm{~m} \mu$ gegen eine Kompensationslösung, die $10 \mathrm{~cm}^{3}$ destilliertes Wasser enthält, sonst jedoch gleich wie die Probe bereitet wird, gemessen werden. Zeigt das zu untersuchende Wasser eine starke Eigenfärbung, so kann der bei der Reaktion entstandene Farbstoff mit Butylalkohol extrahiert werden.

Die Farbreaktion wird nur durch $\mathrm{CN}^{-}$und $\mathrm{CNS}^{-}$hervorgerufen. Erfassungsgrenze: $0,02 \mathrm{mg} \mathrm{CN} / 1$. Wir haben mit der gleichen Reaktion Bestimmungen von Chlorzyan durchgefüht. ASMUS (I952) und auch andere haben auf der gleichen Basis eine Bestimmung von «freiem Chlor» ausgearbeitet.

\section{c) Bestimmung des Gesamtzyanids}

Mit Hilfe der oben beschriebenen titrimetrischen und kolorimetrischen Verfahren wird nur das freie Zyanid und das in schwachen Komplexen gebundene Zyanid erfasst. Es ist aber oft erforderlich, den totalen Zyanidgehalt zu bestimmen. Zu diesem Zwecke muss der Metallkomplex zer- 
stört werden, was am einfachsten durch starkes Ansäuern der Lösung und Abdestillation der dabei gebildeten Blausäure geschehen kann. Bei sehr stabilen Komplexen kann die Zersetzung der Zyanometallyerbindungen beschleunigt werden, wenn zu der Lösung noch andere, das Metall bindende Komplexbildner, wie zum Beispiel Zitronensäure oder Äthylendiamintetraazetat (Komplexon III), zugesetzt werden. Die Tetrazyanokadmiat- und Tetrazyanozinkatkomplexe sind ziemlich unstabil, und das in diesen Verbindungen gebundene Zyanid kann ohne Schwierigkeiten wie «freies» Zyanid mit Silbernitrat titriert werden. Bei den anderen Zyanometallverbindungen wird folgendermassen verfahren: $\sim 100 \mathrm{~cm}^{3}$ der auf Zyanid zu untersuchenden Lösung werden in einen mit Tropftrichter versehenen Destillierkolben gegeben. Diese Lösung versetzt man mit rund 3-4 g Zitronensäure (oder etwa $2 \mathrm{~g}$ Komplexon III). Die Blausäure wird in einer mit Natronlauge gefüllten Vorlage absorbiert. Pro Ioo $\mathrm{mg} \mathrm{CN}^{-}$sind etwa $5 \mathrm{~cm}^{3}$ einer Ioprozentigen Natronlauge vorzulegen. Während der Destillation wird langsam und in kleinen Portionen 20-40prozentige Schwefelsäure aus dem Tropftrichter zur destillierenden Lösung zugesetzt. Die Destillation wird während mindestens 20 min fortgesetzt. Das in der Vorlage absorbierte $\mathrm{CN}^{-}$kann mit Silbernitrat unter Verwendung von Diphenylaminobenzylidenrhodanin als Indikator titriert werden.

Es ist uns im Gegensatz zu BIEG (1948) ohne Schwierigkeiten gelungen, aus sämtlichen untersuchten Zyanometallverbindungen das totale $\mathrm{CN}^{-}$ quantitativ zu erfassen. Einige Beispiele unserer Bestimmungen finden sich in Tabelle 3 .

Tabelle 3

\begin{tabular}{|c|c|c|c|}
\hline $\begin{array}{c}\text { Untersuchter Komplex } \\
\text { stöchiometrischer } \\
\text { Zusammensetzung }\end{array}$ & $\begin{array}{c}\text { Destillationsdatier } \\
\text { in Minuten }\end{array}$ & $\begin{array}{c}\text { (CN) } \\
\text { Sollat } \\
\text { mg/l }\end{array}$ & $\begin{array}{c}\text { gefunden } \\
\text { mg/l }\end{array}$ \\
\cline { 2 - 4 } & & 317 & 316 \\
$\mathrm{Na}_{2}\left[\mathrm{Cd}(\mathrm{CN})_{4}\right]$ & ohne Destillation & 9,2 & 9,1 \\
$\mathrm{~K}_{4}\left[\mathrm{Fe}(\mathrm{CN})_{6}\right]$ & 20 & 462 & 462 \\
$\mathrm{~K}_{3}\left[\mathrm{Fe}(\mathrm{CN})_{6}\right]$ & 20 & 272 & 267 \\
$\mathrm{~K}_{2}\left[\mathrm{Hg}(\mathrm{CN})_{4}\right]$ & 15 & 408 & 406 \\
$\mathrm{~K}_{2}\left[\mathrm{Ni}(\mathrm{CN})_{4}\right]$ & 10 & & \\
\hline
\end{tabular}




\section{BIOLOGISCHE TESTVERSUCHE}

Gemäss den eingangs dargelegten Absichten sind wir von unserem herkömmlichen Schema fischtoxikologischer Untersuchungen abgewichen und haben uns zur Prüfung der entgifteten Zyanidlösungen auf einen einfachen Fischtest beschränkt. Für die Darstellung der Ergebnisse genügt deshalb die Wiedergabe der tabellarisch zusammengezogenen Versuchsprotokolle. Zum Vergleich mit der Wirkung reiner Blausäure haben wir die aus früheren Arbeiten durch Extrapolation ermittelten entsprechenden Werte herangezogen.

Die Tabelle 4 zeigt:

a) Bei der Oxydation von $\mathrm{CN}^{-}$-haltigen Lösungen zu Zyanat tritt eine verblüffende Entgiftungswirkung ein (vgl. II und III). Hingegen darf nicht übersehen werden, dass Zyanationen in grösserer Konzentration eine schädigende Wirkung ausüben können. Ferner ist zu beachten, dass in konzentrierten Lösungen entsprechend der Reaktion ( $3 b$ ) Ammoniak entstehen kann.

b) Die mit Javellewasser oxydierten $\mathrm{CN}^{-}$-Lösungen dürfen kein Chlorzyan enthalten. Lösungen, in denen $\mathrm{ClCN}$ deutlich nachgewiesen werden kann, erweisen sich als schädlicher als reine, unbehandelte Zyanidlösungen entsprechender Konzentration.

c) Alle Versuchstiere wurden nach der Behandlung noch während mehrerer Tage beobachtet. Sie schienen völlig normal und haben offenbar keine wesentliche Störung lebenswichtiger Funktionen erlitten.

Da die Abwässer aus der galvanotechnischen Industrie meistens Schwermetallsalze ( $\mathrm{Cd}, \mathrm{Zn}, \mathrm{Cu}, \mathrm{Ni}, \mathrm{Fe})$ enthalten, war zu untersuchen, ob auch dem komplex gebundenen Zyanid Giftwirkung zukomme. Die Beantwortung dieser Frage gibt uns Aufschluss darüber, ob das komplex gebundene Zyanid durch Javellelauge oxydiert werden muss. In Tabelle $s$ haben wir deshalb die Wirkung von Lösungen verschiedener Zyanometallkomplexe miteinander verglichen.

Wenn das an Schwermetallionen gebundene Zyanid mit Javellewasser oxydiert wird, fallen reine Metallsalzlösungen an. $\mathrm{Ob}$ auch ihnen gewisse fischgiftige Eigenschaften zuzuschreiben sind, haben wir noch in einigen Ergänzungsversuchen, die ebenfalls in Tabelle 5 wiedergegeben sind, abzuklären versucht. 
Tabelle 4

Toxikologische Überprïfung der Zyanid«entgiftung》 mit Hypocblorit

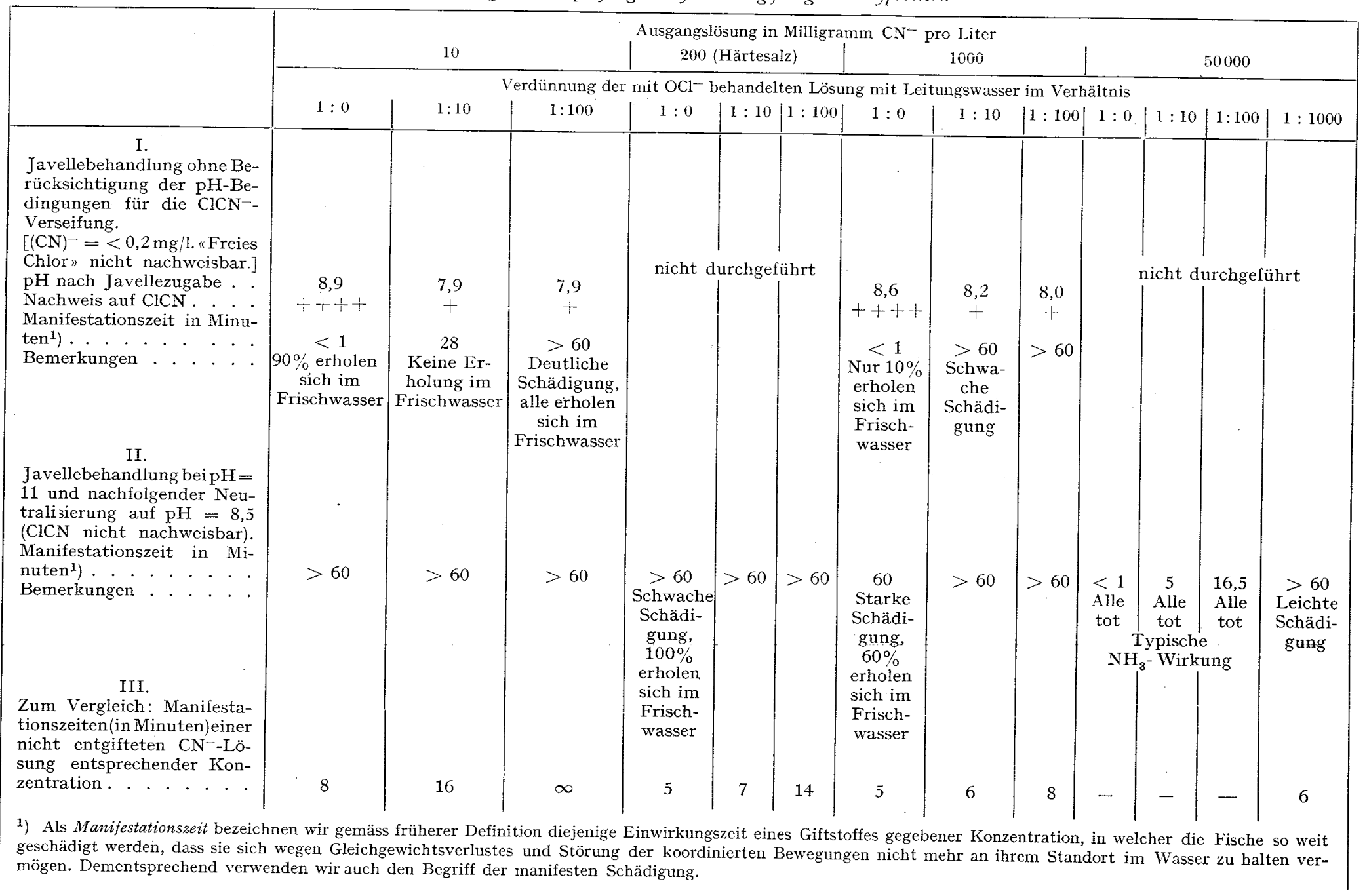


Tabelle 5

Toxikologische Überprïfung von Lösungen, die Zyanometallkomplexe enthalten

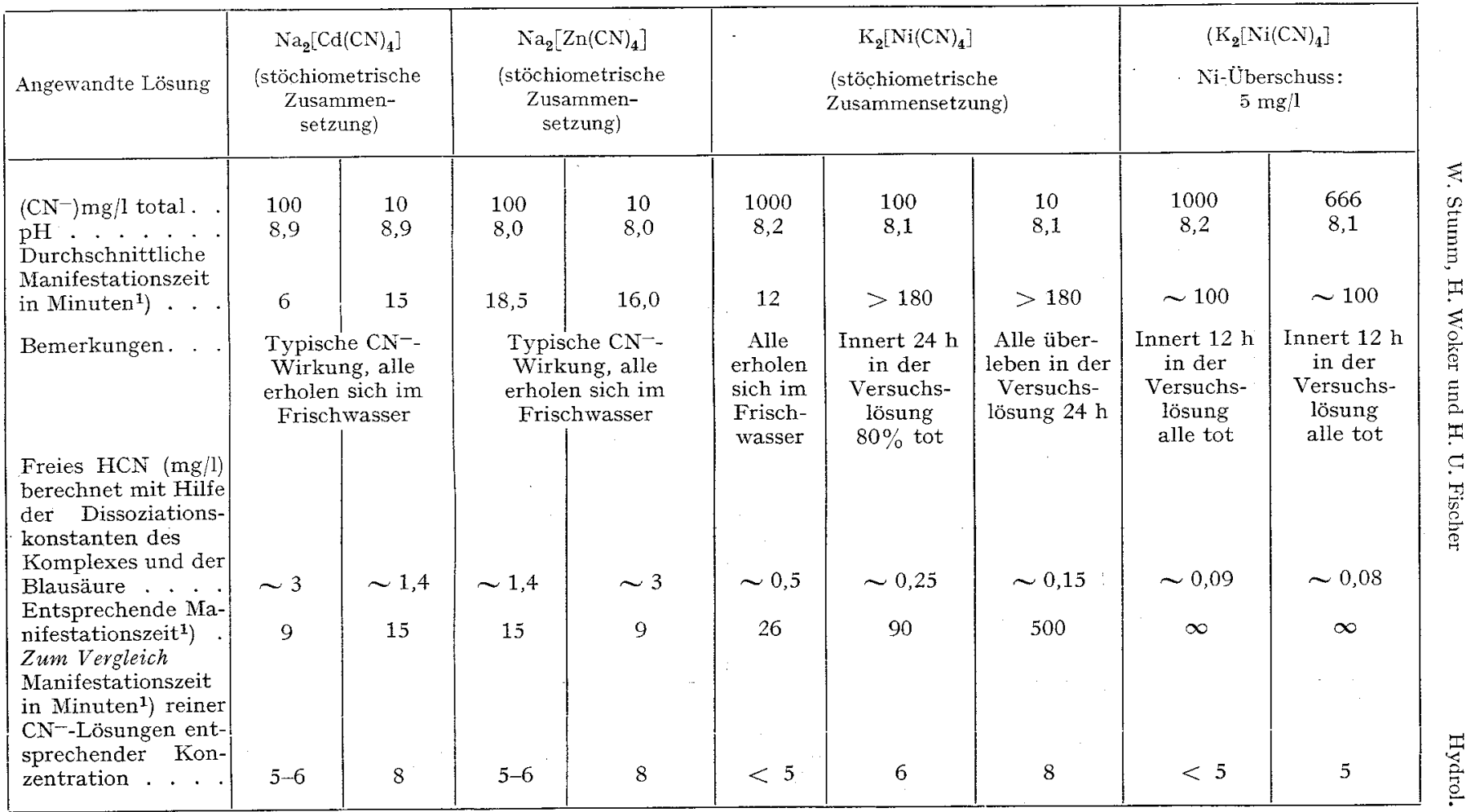




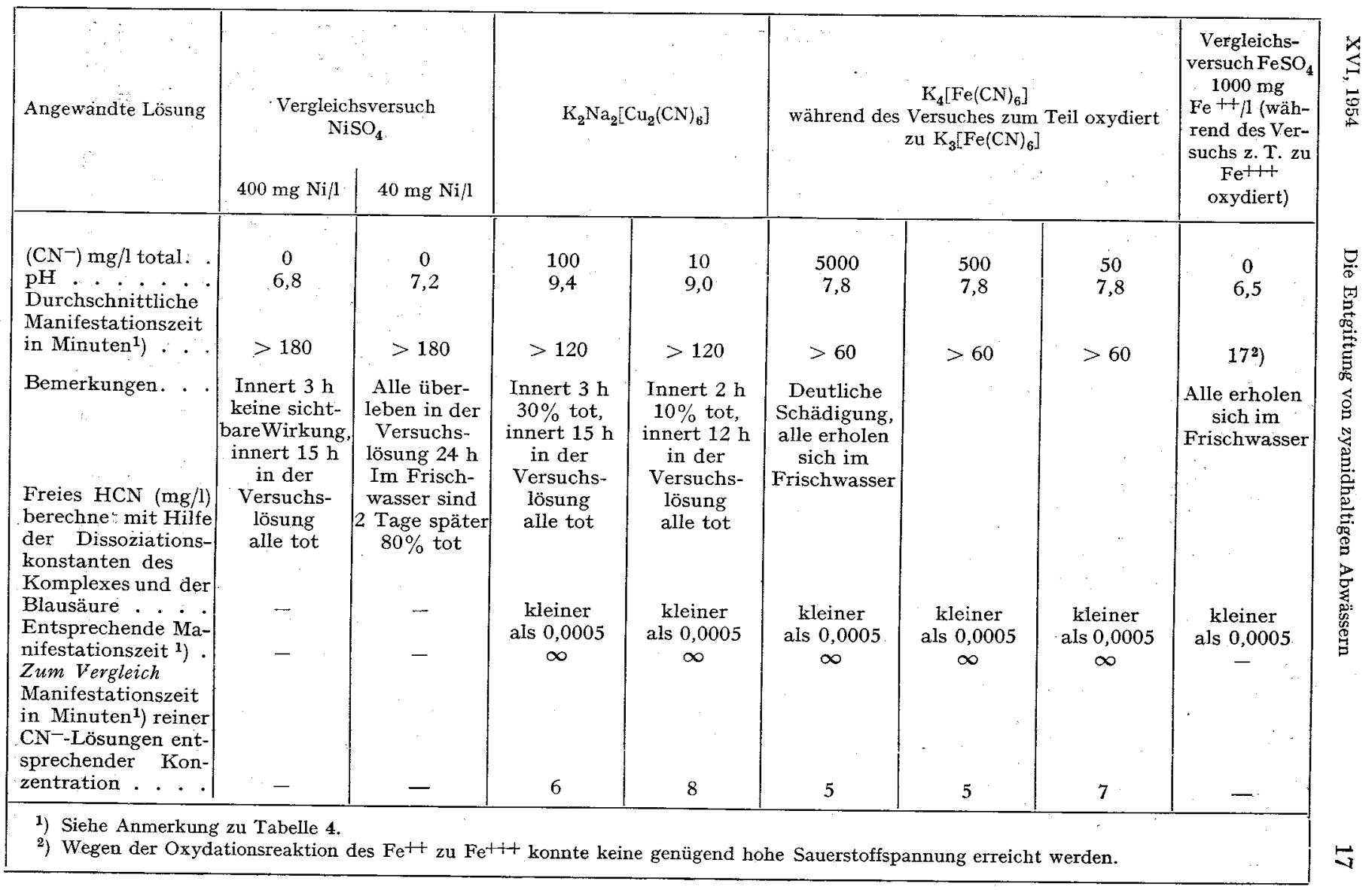


Die in Tabelle $s$ aufgeführten Ergebnisse zeigen:

a) Die Giftwirkung von Lösungen, die Zyanometallkomplexe enthalten, nimmt mit abnehmender Stabilität der entsprechenden komplexen Verbindungen zu. So sind die relativ unstabilen Tetrazyanokadmiat- und Tetrazyanozinkatlösungen in ihrer Giftigkeit gegenüber reinen Zyanidlösungen von korrespondierendem Gehalt an freiem $\mathrm{CN}^{-}$nur geringfügig herabgesetzt. Dagegen erweisen sich die wesentlich stabileren Kupfer-, Nickel- und Eisenkomplexe als verhältnismässig weniger schädlich. So enthält beispielsweise eine Lösung von $\left[\mathrm{Cd}(\mathrm{CN})_{4}\right]^{--}$der Konzentration $\mathrm{cCN}^{-}{ }_{\text {total }}=10 \mathrm{mg} / \mathrm{l}$ zirka $\mathrm{I}, 4 \mathrm{mg} \mathrm{HCN} / \mathrm{l}$. Die durchschnittliche Manifestationszeit ${ }^{1}$ ) für I,4 $\mathrm{mg} \mathrm{HCN} / 1$ beträgt Is min, was sich mit der experimentell am Kadmiumkomplex ermittelten Manifestationszeit ( I $5 \mathrm{~min}$ ) deckt. Hiebei ist allerdings zu berücksichtigen, dass diese Übereinstimmung im Experiment nicht immer so genau erreicht werden kann, da es ausserordentlich schwerfällt, Komplexe von genauer stöchiometrischer Zusammensetzung herzustellen. Sicher zeigen aber unsere Versuche eindeutig (im Gegensatz zur Auffassung von MEINCK, 1942), dass in der toxischen Wirkung ein eminenter Unterschied zwischen komplex gebundenem und freiem Zyanid besteht.

b) Unsere Versuche haben gezeigt, dass stabile Zyanokomplexe relativ ungiftig sind. Anderseits geht aus Tabelle 5 hervor, dass reinen Metallsalzlösungen eine gewisse Giftwirkung zukommt. Aus diesem Grunde ist es nicht notwendig und unter Umständen sogar unerwünscht, das in stabilen Komplexen gebundene Zyanid durch Javellewasser zu oxydieren, weil dadurch unnötigerweise die Konzentration an freien Metallionen erhöht wird. Es erweist sich als Vorteil, dass wir analytisch unterscheiden können zwischen stark und schwach komplex gebundenem Zyanid, das heisst, wir können durch Titration mit $\mathrm{AgNO}_{3}$ die relative Giftigkeit der Zyankomplexe erfassen. Dementsprechend ist es notwendig, nur das in der direkten Titration mit Silbernitrat erfassbare Zyanid durch Oxydation zu zerstören.

Ein Vergleich zwischen den Tabellen 4 und 5 zeigt, dass durch die stark komplexe Bindung des Zyanids an Schwermetalle wohl die typische Zyanidwirkung weitgehend eliminiert wird, dass aber trotzdem bei längerer Einwirkungszeit Schädigungen auftreten können. Insbesondere ist ein eventueller Einfluss von «freien» Schwermetallionen im Auge zu behalten. So brachte gerade die zum Teil bis auf $24 \mathrm{~h}$ ausgedehnte toxikologische

1) Siehe Anmerkung zu Tabelle 4. 
Prüfung dieser Zyanometallkomplexe den Beweis, dass die Fische bei längerer Einwirkungszeit unter Umständen doch getötet werden können. Von besonderem Interesse scheint diesbezüglich die Beobachtung an den mit reinem Nickelsulfat behandelten Tieren, die sich nach 24stündiger Versuchsdauer - in Frischwasser umgesetzt - scheinbar rasch erholten, zwei Tage später aber doch noch eingingen. Diese typische Spätwirkung ist ein deutlicher Hinweis auf die Gefährlichkeit von Schwermetallionen im Abwasser.

Zusammenfassend darf man also festhalten, dass uns im Rahmen des begrenzten Gültigkeitsbereiches der angewendeten biologischen Teste der Nachweis einer wirksamen Entgiftung von Zyanidlösungen mit Javellelauge gelungen ist. Das Verfahren ist jedoch nur dann anwendbar, wenn die zyanidhaltigen Abwässer weniger als $\mathrm{I} \mathrm{g} \mathrm{CN}^{-} / 1$ enthalten. Auch unter dieser Voraussetzung sollten die entgifteten Lösungen in der Vorflut aus Sicherheitsgründen noch mindestens eine Verdünnung im Verhältnis I: Io erfahren.

In Anlehnung an unsere übrigen fischtoxikologischen Arbeiten haben wir auch für die vorliegende Untersuchung Elritzen als Testfische gewählt. Es stellt sich nun die Frage nach dem allgemeinen Gültigkeitsbereich der gewonnenen Erkenntnisse. Wir haben früher einen Vergleich der Empfindlichkeit von Elritzen und anderen Fischarten gegenüber Zyanid- bzw. Blausäurelösung angestellt (WUHRMANN und WOKER, I952 und 1953). Daraus geht hervor, dass Elritzen auf freies Zyanid zum Teil wesentlich empfindlicher reagieren als beispielsweise Schleien und Alet und dass ihre Konzentrations-Wirkungs-Funktion ungefähr derjenigen des Flussbarsches entspricht. Auf Grund der von HERBERT und MERKENS (1952) publizierten Ergebnisse muss angenommen werden, dass Regenbogenforellen aber empfindlicher ansprechen. Wir sind jedoch der Auffassung, dass die nach unserer Vorschrift behandelten Zyanidbäder trotzdem in Salmonidengewässer abgeleitet werden dürfen, da die geringe Giftwirkung der entstehenden Lösungen und die postulierten Einleitungsbedingungen (Verdünnung I: Io in der Vorflut) auch für Forellen genügend Sicherheit bieten.

\section{ENTGIFTUNGSVORSCHRIFT}

Auf Grund unserer Untersuchungen sind wir nun in der Lage, eine Vorschrift für die Entgiftung zyanidhaltiger Abwässer, die Konzentrationen von höchstens Iooo $\mathrm{mg} \mathrm{CN}^{-} / 1$ aufweisen, vorzuschlagen: 
I. In $100 \mathrm{~cm}^{3}$ der zu behandelnden Lösung ist durch Titration mit Silbermety $/$ nitrat (unter Verwendung von D laminobenzylidenrhodanin als Indikator) die Konzentration des freien und des schwach komplex gebundenen Zyanids, welche für die Giftwirkung verantwortlich ist, $\mathrm{zu}$ ermitteln ( $\mathrm{I} \mathrm{cm}^{3} \mathrm{~m} / \mathrm{Io} \mathrm{AgNO}_{3}$ entspricht $5,2 \mathrm{mg} \mathrm{CN}$ ).

2. Mit Universalindikatorpapier (zum Beispiel Merk) ist zu prüfen, ob die Lösung genügend alkalisch ist. (Das Indikatorpapier muss eine grünblaue oder blaue Färbung aufweisen.) Ansonst muss man der Lösung technische Natronlauge, Soda oder Kalkmilch zufügen.

3. Pro Gramm Zyanid ( $\mathrm{CN}^{-}$) sind 2,73 g freies Chlor ( $18,2 \mathrm{~cm}^{3}$ einer Is prozentigen Javellelauge) langsam und unter Umrühren zuzugeben, wobei $\mathrm{zu}$ vermeiden ist, dass die Temperatur des Bades $50^{\circ} \mathrm{C}$ übersteigt.

4. Nach der Javellezugabe muss die behandelte Lösung noch während IO-I 5 min umgerührt werden.

5. Nach rund einer halben Stunde wird der Zyanidgehalt des Bades erneut, wie unter $\mathrm{I}$ beschrieben, mit Silbernitrat titriert, wobei dieses Mal vorteilhafterweise $500-1000 \mathrm{~cm}^{3}$ der Lösung zur Analyse gelangen. Wiederum wird, wie unter 3 und 4 ausgeführt, entsprechend der jetzt noch vorhandenen Zyanidmenge Javellelauge zugegeben.

6. Die unter 5 beschriebenen Operationen müssen so oft wiederholt werden, bis die Konzentration an titrierbarem Zyanid kleiner als $0,2 \mathrm{mg}$ $\mathrm{CN}^{-} / 1$ ist.

7. Das behandelte Bad muss nun einige Zeit stehen. Die Wartefrist richtet sich nach der Alkalität $(\mathrm{pH})$, die die Lösung jetzt aufzeigt:

Farbe des Universal-Indikatorpapiers "Merk»:

$$
\begin{aligned}
& \text { grünlich } \\
& \text { grünlichblau } \\
& \text { blau }
\end{aligned}
$$

Wartefrist mindestens:

$$
\begin{array}{r}
20 \mathrm{~h} \\
\mathrm{I} 2 \mathrm{~h} \\
3-4 \mathrm{~h}
\end{array}
$$

8. Hat die Lösung nach dem Stehenlassen einen $\mathrm{pH}$, der höher als 9 liegt (Indikatorpapier = blau), so muss langsam und unter Umrühren Säure zugefügt werden. Die Konzentration der Säure (Salzsäure, Schwefelsäure) darf höchstens sprozentig sein. Es wird so lange Säure zugegeben, bis ein $\mathrm{pH}$ von 8,5-9 (Indikatorpapier = grünblau; der Indikator darf auf keinen Fall rot werden) erreicht wird.

9. Auf diese Weise entgiftete Bäder sind vor dem Abfliessenlassen mit Io Teilen frischen Wassers zu verdünnen. 


\section{ZUSAMMENFASSUNG}

I. Die chemischen Reaktionen, die bei der Oxydation von zyanidhaltigen Abwässern mit Javellelauge auftreten, werden diskutiert. Durch experimentelle Untersuchungen haben wir diejenigen Bedingungen ermittelt, die für eine erfolgreiche Entgiftung eingehalten werden müssen. In unsere Untersuchungen haben wir auch Lösungen, die Zyanometallkomplexe enthalten, einbezogen. Die Geschwindigkeit der Hydrolyse von Chlorzyan, das bei der Oxydation von Zyanid mit Hypochlorit stets als Zwischenprodukt auftritt, wurde bestimmt.

2. Die zur Untersuchung der chemischen Reaktionen notwendigen analytischen Bestimmungsmethoden $\left(\mathrm{CN}^{-}\right.$, komplex gebundenes $\mathrm{CN}^{-}$, $\mathrm{ClCN}$ ) wurden überprüft.

3. Die an Hand unserer chemischen Untersuchungen ausgearbeiteten Entgiftungsvorschriften wurden biologischen Testversuchen (Elritzen) unterzogen.

4. Eine detaillierte Entgiftungsvorschrift wird gegeben.

\section{LITERATURVERZEICHNIS}

ALDRIDGE, W. N., Analyst 69, 262 (I944).

Asmus, E., Z. anal. Chem. 136, 269 (1952).

BIEG, S., Gesundheitsingenieur 69, 324, 355 (1948).

Epstein, J., Anal. Chemistry 19, 272 (I947).

Herbert, D. W. M., und Merkens, J. C., J. exp. Biol. 29, 632 (1952).

KöNIG, W., J. prakt. Chem. 69, IOS (1904).

MEINCK, F., Metallindustr. Galvanotechn. 40, 225 (1942).

MILNE, D., Sewage ind. Wastes 22, 904, I I92 (I950).

WILl, E. G., Sewage ind. Wastes 23, I288 (I95I).

Woker, H., und WuHrmanN, K., Verh. int. Ver. theor. angew. Limnol. 10, 575

(1949).

WUHR MANN, K., und Woker, H., Schweiz. Z. Hydrol. 11, 210 (I948).

WuhrmanN, K., und Woker, H., Schweiz. Z. Hydrol. 12, 79 (1950).

WuHrmanN, K., Bull. Centre Belge Etude Document. Eaux No Is (1952/I).

WuhrmanN, K., und WOKER, H., in: Fiscb und Fischerei (Schmid, Winterthur 1952).

WuhrmanN, K., und WoKer, H., Schweiz. Z. Hydrol. 15, 236 (I953). 